\title{
NOUVELLe
}

\section{Rôle hépato-protecteur de l'amphiréguline, un ligand du récepteur de l'EGF, en situation de cholestase}

N’Diaye Sidibé ${ }^{1}$, Hadrien Jalabert ${ }^{1}$, Gregory Merlen², Thierry Tordjmann ${ }^{2}$
${ }^{1}$ Master 1 Biologie santé, Université Paris-Saclay, 91405 Orsay, France.

${ }^{2}$ Équipe Homéostasie biliaire et réparation du foie, Inserm U1193, Université Paris-Saclay, 91405 Orsay, France.

ndiaye.sidibe@universite-paris-saclay.fr

gregory.merlen@universite-paris-saclay.fr

thierry.tordjmann@universite-paris-saclay.fr
> La cholangite biliaire primitive (CBP) et la cholangite sclérosante primitive (CSP) sont des maladies inflammatoires chroniques des voies biliaires du foie entraînant un dysfonctionnement de la sécrétion biliaire. Ces maladies sont associées à une cholestase, c'est-àdire à une diminution de la sécrétion de bile [1]. D'autres maladies cholestatiques peuvent être d'origine génétique ou encore acquise (e.g., obstruction des voies biliaires par un calcul). Elles ont pour conséquence une accumulation des acides biliaires ( $A B$ ) dans les cellules hépatiques et dans le sang, entraînant des dommages tissulaires oxydatifs. La pathogenèse de ces maladies, en particulier celle de la CBP et de la CSP, est mal connue. Parmi les acteurs impliqués, le récepteur de l'EGF (epidermal growth factor receptor, $\varepsilon G F R)$ est considéré comme jouant un rôle important [2].

\section{L'EGFR et son ligand, l'amphiréguline (AREG), dans la signalisation hépatique}

L'EGFR est une protéine transmembranaire possédant une activité tyrosine kinase. Ce récepteur intervient notamment dans la prolifération, la différenciation, l'adhérence, la polarité, la migration et la survie cellulaires, en activant différentes voies intracellulaires de signalisation. Ces voies impliquent des kinases (mitogen-activated protein kinases, MAPK, ou phosphoinositide 3-kinase, PI3K), une phospholipase (PLC- $\gamma$ ), et des facteurs de transcription (signal transducer and activator of transcription, STAT) [3]. En dehors de l'EGF, plusieurs autres ligands tels que le TGF- $\beta$ (transforming growth factor $\beta$ ) et l'amphiréguline (AREG), un facteur de croissance connu pour son rôle dans le développement des glandes mammaires et dans le cancer du sein [4], peuvent se lier à I'EGFR avec des affinités différentes.

Dans le foie, la signalisation par l'EGFR est impliquée dans des mécanismes de protection et de réparation, et plus particulièrement au cours de la régénération [5-9]. Dans un article récemment publié, Santamaria et al. ont analysé le rôle de I'AREG en situation de cholestase, ainsi que sa régulation par les $A B$. Ces chercheurs ont analysé l'activation de la voie de l'EGFR et l'expression de I'AREG au cours de la CBP et de la CSP, ainsi que dans des modèles animaux de cholestase, et ont démontré le rôle protecteur de I'AREG [7].

\section{L'expression de I'AREG protège des} lésions hépatiques liées à la cholestase Les auteurs ont d'abord analysé des échantillons de foie de patients atteints de CBP ou de CSP, dans lesquels ils ont trouvé une surexpression de l'AREG. Ils ont ensuite eu recours à deux modèles murins expérimentaux de cholestase : des souris chez lesquelles une cholestase a été induite chirurgicalement, par ligature de la voie biliaire principale (bile duct ligation, BDL), ou chimiquement, en leur administrant par voie orale une substance toxique ( $\alpha$-naphthyl-isothiocyanate, ANIT). Dans le modèle chirurgical de cholestase (BDL), une accumulation des $A B$ entraînant une dégradation du tissu hépatique est observée dans les jours suivant la chirurgie de ligature. Dans le modèle chimique, I'ANIT déclenche une cholestase aiguë intense, avec des lésions des cholangiocytes et des hépatocytes. Dans ces deux modèles murins de cholestase, une surexpression de I'AREG a également été observée. En mesurant chez ces souris, par une analyse histologique, les proportions de tissu hépatique présentant des lésions et en les comparant à celles observées chez des souris rendues génétiquement déficientes en AREG (souris $\mathrm{Areg}^{-/-}$), les auteurs ont constaté que les souris $\mathrm{Areg}^{-/-}$présentaient des lésions hépatiques plus étendues, avec quatre fois plus de foyers nécrotiques que les souris de génotype «sauvage» $\left(\mathrm{Areg}^{+/+}\right)$. Ils ont également montré que l'administration d'AREG chez des souris $\mathrm{Areg}^{+/+}$dans le modèle ANIT de cholestase réduisait les lésions hépatiques.

Afin de quantifier la mort cellulaire dans ces différents groupes de souris, les auteurs se sont appuyés sur l'analyse histologique de la nécrose tissulaire hépatique et sur les dosages sanguins d'enzymes hépatiques, en particulier les transaminases (dont l'augmentation du taux plasmatique est associée à la cytolyse hépatique). Les taux plasmatiques des transaminases, ainsi que ceux de la bilirubine et des phosphatases alcalines, étaient beaucoup plus élevés chez les souris $\mathrm{Areg}^{-/-}$que chez les souris Areg ${ }^{+/+}$. L'analyse des mécanismes cellulaires 


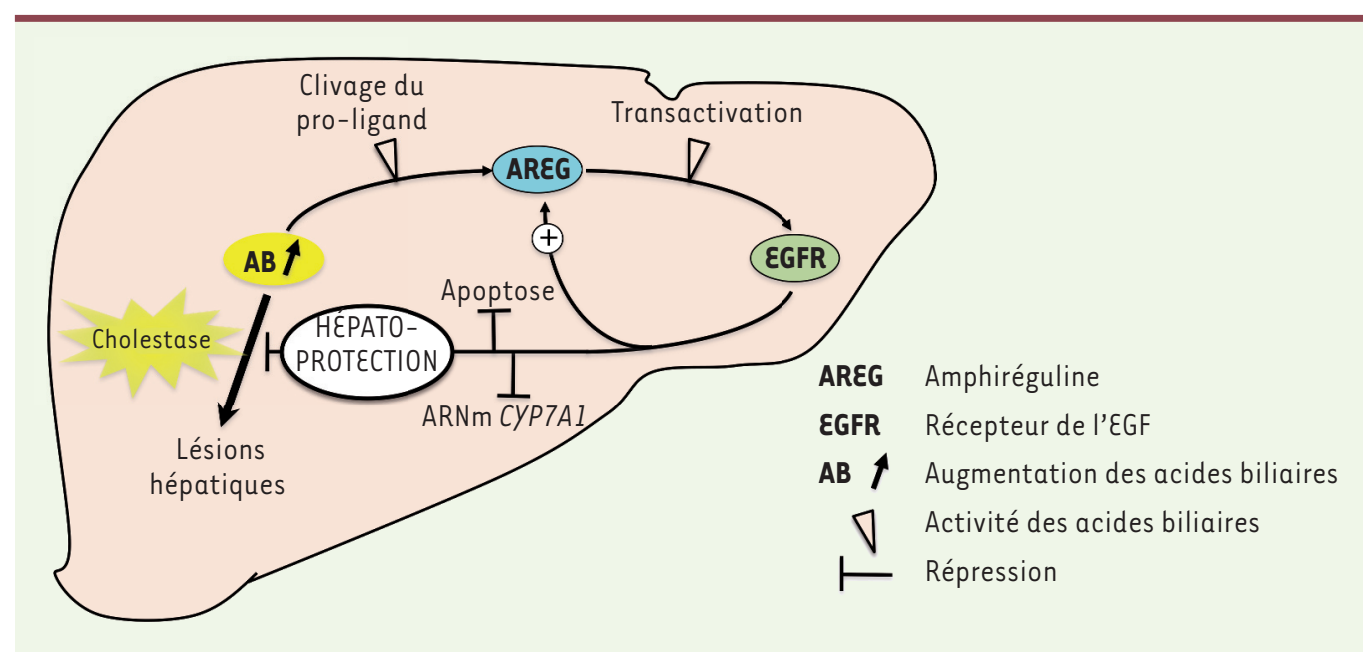

Figure 1. L'amphiréguline protège le foie des lésions associées à la cholestase. En situation de cholestase, les acides biliaires ( $A B$ ) s'accumulent dans le foie et peuvent être à l'origine de lésions tissulaires. Les $A B$ peuvent induire la sécrétion d'amphiréguline (AREG) par clivage protéolytique de la pro-amphiréguline membranaire.

L'AREG active le récepteur de l'EGF (EGFR), qui déclenche des voies anti-apoptotiques et une inhibition de la synthèse des $A B$ par répression des ARNm de la cholestérol 7- $\alpha$-monooxygénase (CYP7Al), enzyme limitante de la synthèse des AB. L'activation de l'EGFR entraîne également une induction du gène codant l'AREG. Tous ces mécanismes contribuent à protéger le foie contre les effets délétères de la surcharge en $A B$ en situation de cholestase.

impliqués a montré que l'AREG agit comme une molécule anti-apoptotique, qui protège donc des lésions induites par la cholestase en empêchant la mort cellulaire. Ces résultats obtenus in vivo ont été confirmés par des expériences in vitro. $\varepsilon n$ traitant des hépatocytes murins avec I'AREG, les auteurs ont observé une inhibition de l'effet pro-apoptotique des $A B$. Plus précisément, l'analyse par western blot a montré qu'en présence d'AB primaires, tels que l'acide chénodésoxycholique ( $C D C A)$, ou d'AB secondaires, tels que l'acide lithocholique (LCA), I'AREG inhibe le clivage de la caspase 3 , qui est une étape essentielle de l'apoptose cellulaire. En plus de cette action antiapoptotique de I'AREG, les auteurs ont mis en évidence une régulation mutuelle entre l'expression du gène de l'AREG et la synthèse des $A B$ (Figure 1). En effet, les résultats présentés indiquent que I'AREG est capable d'inhiber physiologiquement la synthèse des $A B$. Chez la

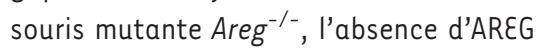
entraîne : 1) une augmentation des ARNm de la cholestérol 7- $\alpha$-monooxygénase hépatocytaire (CYP7Al), qui est l'enzyme limitante impliquée dans la transformation du cholestérol en $A B[10,11] ; 2$ ) une élévation des concentrations en $A B$ dans les différents compartiments du cycle entéro-hépatique (plasma, foie et fèces) ; et 3) une réduction du cholestérol plasmatique. Inversement, l'administration d'un repas-test (un gavage avec une solution riche en nutriments) chez les souris $\mathrm{Areg}^{+/+}$in vivo, ou bien la stimulation in vitro des hépatocytes avec de l'AREG, fait chuter les ARN messagers (ARNm) de CYP7Al par des mécanismes impliquant les voies MAPK/ERK (extracellular signal-regulated kinase) et JNK (c-Jun N-terminal kinase).

\section{Signalisation par l'AREG}

Cette étude détaillée des mécanismes reliant $A B, A R \varepsilon G$ et $\varepsilon G F R$ a révélé l'existence de multiples voies de régulations réciproques entre ces acteurs moléculaires. D'une part, les $A B$ induisent le clivage de la pro-AREG membranaire en activant la métalloprotéase ADAM17, ce qui induit la libération d'AREG dans le milieu extracellulaire. L'AREG va ensuite activer I'EGFR membranaire, ce qui constitue un stimulus puissant pour la synthèse d'AREG par induction de son ARNm (feed-back positif). D'autre part, les $A B$ induisent la synthèse d'EGFR par un mécanisme indirect dépendant du récepteur nucléaire des $A B, F X R$ (farnesoid $X$ receptor). En effet, les auteurs ont mis en évidence que les $A B$ régu- laient positivement l'expression des ARNm de l'AREG et de l'EGFR, de façon au moins en partie dépendante de FXR.

\section{Conclusion et perspectives}

Dans cette étude, les auteurs ont démontré le rôle hépato-protecteur de I'AREG activant la signalisation par l'EGFR. L'effet de cette signalisation complexe combine une action antiapoptotique s'opposant aux effets délétères de la surcharge en $A B$, une inhibition de la synthèse des $A B$, ainsi qu'une augmentation de la synthèse d'EGFR (Figure 1). Il existe par ailleurs d'autres mécanismes hépato-protecteurs induits par les $A B$ eux-mêmes (réponses adaptatives) et impliquant leur récepteur nucléaire FXR et leur récepteur membranaire TGR5 (Takeda G protein-coupled receptor 5) [12]. Ces différentes voies de signalisation aux effets hépato-protecteurs constituent autant de cibles thérapeutiques potentielles au cours des maladies cholestatiques. Leur interaction entraîne l'activation d'une voie qui module physiologiquement la synthèse des $A B$ durant le rythme alimentaire, et qui est également puissamment hépatoprotectrice en situation de cholestase. The EGFR ligand amphiregulin protects from cholestatic liver injury 


\section{LIENS D'INTÉRÊT}

Les auteurs déclarent n'avoir aucun lien d'intérêt concernant les données publiées dans cet article.

\section{RÉFÉRENCES}

1. Jansen PLM, Ghallab A, Vartak N, et al. The ascending pathophysiology of cholestatic liver disease. Hepatology $2017 ; 65$ : 722-38.

2. Svinka J, Pflügler S, Mair M, et al. Epidermal growth factor signaling protects from cholestatic liver injury and fibrosis. J Mol Med 2016; 95 : 109-17.

3. Wieduwilt MJ, Moasser MM. The epidermal growth factor receptor family: Biology driving targeted therapeutics. Cell Mol Life Sci 2008 ; 65 : 1566-84.
4. Berasain C, Avila MA. Amphiregulin. Semin Cell Dev Biol $2014 ; 28: 31-41$.

5. Berasain C, García-Trevijano ER, Castillo J, et al. Amphiregulin: an early trigger of liver regeneration in mice. Gastroenterology $2005 ; 128$ : 424-32.

6. Berasain C, Avila MA. The EGFR signalling system in the liver: from hepatoprotection to hepatocarcinogenesis. J Gastroenterol $2013 ; 49: 9-23$.

7. Santamaría $\varepsilon$, Rodríguez Ortigosa CM, Uriarte I, et al. The epidermal growth factor receptor ligand amphiregulin protects from cholestatic liver injury and regulates bile acids synthesis. Hepatology 2019; $69: 1632-47$

8. Collin de l'Hortet A, Gilgenkrantz H, Guidotti JE. EGFR: a master piece in $\mathrm{Gl} / \mathrm{S}$ phase transition of liver regeneration. Int J Hepatol $2012 ; 2012$ : 476910.
9. Natarajan A, Wagner B, Sibilia M. The EGF receptor is required for efficient liver regeneration. Proc Natl Acad Sci USA 2007 ; 104 : 17081-6.

10. Chiang JYL, Ferrell JM. Bile acid metabolism in liver pathobiology. Gene Express 2018 ; 18 : 71-87.

11. Russell DW. The enzymes, regulation, and genetics of bile acid synthesis. Ann Rev Biochem 2003; 72 : $137-74$.

12. Merlen G, Bidault-Jourdainne V, Kahale N, et al. Hepatoprotective impact of the bile acid receptor TGR5. Liver Int 2020 ; 40 : 1005-15.

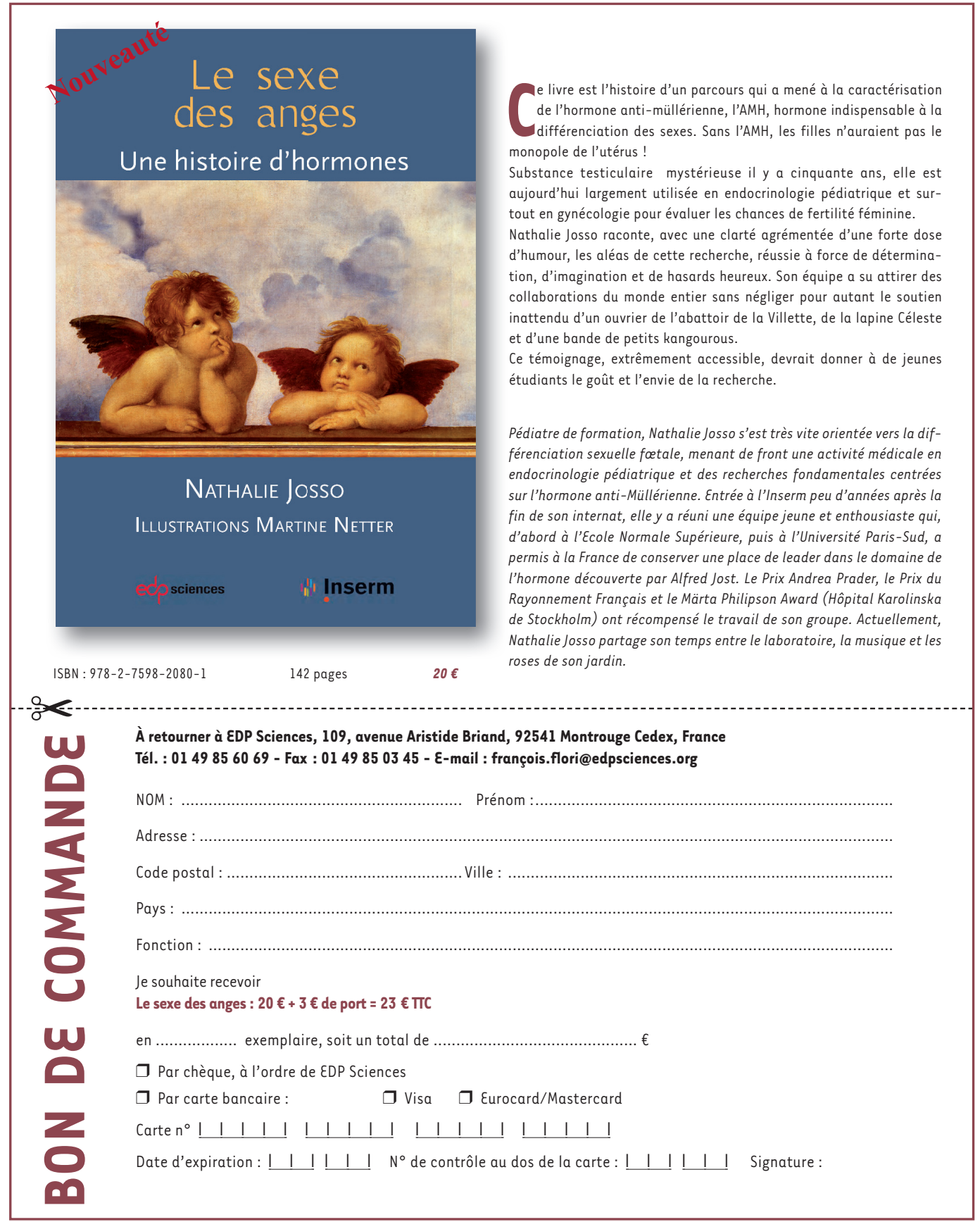

